\title{
ON GLOBAL ROUGH SOLUTIONS TO A NON-LINEAR SCHRÖDINGER SYSTEM
}

\author{
LI MA \\ Department of Mathematical Sciences, Tsinghua University, Peking 100084, P.R. China \\ e-mail:lma@math.tsinghua.edu.cn \\ XIANFA SONG \\ Department of Mathematics, Tianjin University, Tianjin 300072, P.R. China \\ e-mail: songxianfa2004@163.com \\ and LIN ZHAO \\ Department of Mathematical Sciences, Tsinghua University, Beijing, P.R. China \\ e-mail: zhaolin05@mails.tsinghua.edu.cn
}

(Received 1 July 2008; accepted 20 November 2008)

\begin{abstract}
The non-linear Schrödinger systems arise from many important physical branches. In this paper, employing the ' $I$-method', we prove the global-in-time well-posedness for a coupled non-linear Schrödinger system in $H^{s}\left(\mathbb{R}^{n}\right)$ when $n=2$, $s>4 / 7$ and $n=3, s>5 / 6$, respectively, which extends the results of J. Colliander, M. Keel, G. Staffilani, H. Takaoka and T. Tao (Almost conservation laws and global rough solutions to a nonlinear Schrödinger equation, Math Res. Lett. 9, 2002, 659-682) to the system.
\end{abstract}

AMS Classification. Primary 35Q55.

1. Introduction. In this paper, we consider the following Cauchy problem of a non-linear Schrödinger system:

$$
\left\{\begin{array}{l}
i \partial_{t} u_{1}+\Delta u_{1}=\mu_{1}\left|u_{1}\right|^{2} u_{1}+\beta\left|u_{2}\right|^{2} u_{1}, \quad x \in \mathbb{R}^{n}, \quad t>0 \\
i \partial_{t} u_{2}+\Delta u_{2}=\mu_{2}\left|u_{2}\right|^{2} u_{2}+\beta\left|u_{1}\right|^{2} u_{2}, \quad x \in \mathbb{R}^{n}, \quad t>0 \\
u_{1}(x, 0)=u_{10}(x) \in H^{s}\left(\mathbb{R}^{n}\right) \quad \text { and } \quad u_{2}(x, 0)=u_{20}(x) \in H^{s}\left(\mathbb{R}^{n}\right)
\end{array}\right.
$$

Model (1) has applications in many physical problems, especially in non-linear optics. There have been many articles concerning this model in the literature, and here we cite only $[\mathbf{1}, \mathbf{4}, \mathbf{5}, \mathbf{9}, \mathbf{1 0}]$ as references. Recently, the first author and the third author of this paper have obtained some results about the ground state or blow-up solutions of system (1) (see [11-13]).

From the physical angle, a very interesting question is whether the global-in-time well-posedness holds true in some sense when the initial data enjoys infinite energy. In the pioneering paper [6], Colliander, Staffilani, Tao, and the co-authors obtained the global well-posedness for the single Schrödinger equation in $H^{s}\left(\mathbb{R}^{n}\right)$. The aim of this paper is to extend their results to the coupled non-linear Schrödinger system, exploring the method given in [6]. 
Let us recall some corresponding classical results (see $[7,8,11,14,15])$ about the Schrödinger system in $H^{1}\left(\mathbb{R}^{n}\right)$. The mass conservation law about (1) reads

$$
\left\|u_{1}(t)\right\|_{L^{2}\left(\mathbb{R}^{n}\right)}+\left\|u_{2}(t)\right\|_{L^{2}\left(\mathbb{R}^{n}\right)} \equiv\left\|u_{10}\right\|_{L^{2}\left(\mathbb{R}^{n}\right)}+\left\|u_{20}\right\|_{L^{2}\left(\mathbb{R}^{n}\right)},
$$

and the energy conservation law is

$$
\begin{aligned}
E\left(u_{1}, u_{2}\right)(t)= & \int_{\mathbb{R}^{n}} \frac{1}{2}\left(\left|\nabla u_{1}\right|^{2}+\left|\nabla u_{2}\right|^{2}\right) d x \\
& +\frac{1}{4} \int_{\mathbb{R}^{n}}\left(\mu_{1}\left|u_{1}\right|^{4}+\mu_{2}\left|u_{2}\right|^{4}+2 \beta\left|u_{1}\right|^{2}\left|u_{2}\right|^{2}\right) d x \\
\equiv & E\left(u_{1}, u_{2}\right)(0) .
\end{aligned}
$$

Throughout this paper, we make the following assumption on $\mu_{1}, \mu_{2}$ and $\beta$ that the matrix:

$$
\left[\begin{array}{cc}
\mu_{1} & \min \{\beta, 0\} \\
\min \{\beta, 0\} & \mu_{2}
\end{array}\right]
$$

is positive definite. To investigate the global well-posedness in $H^{s}\left(\mathbb{R}^{n}\right)$ instead of $H^{1}\left(\mathbb{R}^{n}\right)$, we need to introduce a modification of the energy functional which is 'almost conserved'. Given $s<1$ and a parameter $N \gg 1$, we define the multiplier operator

$$
\widehat{I_{N} f}(\xi)=M_{N}(\xi) \hat{f}(\xi)
$$

where the multiplier $M_{N}(\xi)$ is smooth, radially symmetric, non-increasing in $|\xi|$ and

$$
M_{N}(\xi)=\left\{\begin{array}{l}
1, \quad|\xi| \leq N \\
\left(\frac{N}{|\xi|}\right)^{1-s}, \quad|\xi| \geq 2 N
\end{array}\right.
$$

Note that

$$
\begin{aligned}
E\left(I_{N} u_{1}, I_{N} u_{2}\right)(t) \leq & N^{2-2 s}\left(\left\|u_{1}(\cdot, t)\right\|_{\dot{H}^{s}\left(\mathbb{R}^{n}\right)}^{2}+\left\|u_{2}(\cdot, t)\right\|_{\dot{H}^{s}\left(\mathbb{R}^{n}\right)}^{2}\right) \\
& +\mu_{1}\left\|u_{1}(\cdot, t)\right\|_{L^{4}\left(\mathbb{R}^{n}\right)}^{4}+\mu_{2}\left\|u_{2}(\cdot, t)\right\|_{L^{4}\left(\mathbb{R}^{n}\right)}^{4}+2|\beta|\left\|u_{1}(\cdot, t) u_{2}(\cdot, t)\right\|_{L^{2}\left(\mathbb{R}^{n}\right)}^{2},
\end{aligned}
$$

$$
\begin{aligned}
& \left\|u_{1}(\cdot, t)\right\|_{H^{s}\left(\mathbb{R}^{n}\right)}^{2}+\left\|u_{2}(\cdot, t)\right\|_{H^{s}\left(\mathbb{R}^{n}\right)}^{2} \\
& \leq C E\left(I_{N} u_{1}, I_{N} u_{2}\right)(t)+C\left\|u_{10}\right\|_{L^{2}\left(\mathbb{R}^{n}\right)}^{2}+C\left\|u_{20}\right\|_{L^{2}\left(\mathbb{R}^{n}\right)}^{2} .
\end{aligned}
$$

To prove the global well-posedness, it is equivalent to obtain a bound on the $H^{s}$-norm of the solution which grows at most polynomially in $t$. To be special, we intend to prove

$$
E\left(I_{N} u_{1}, I_{N} u_{2}\right)(t) \preceq(1+t)^{L},
$$

for a positive constant $N$ depending on $t$ and a positive constant $L$ depending on $\left\|u_{10}\right\|_{H^{s}\left(\mathbb{R}^{n}\right)}+\left\|u_{20}\right\|_{H^{s}\left(\mathbb{R}^{n}\right)}$. The assumption that matrix (4) is positive definite guarantees that estimate (9) implies the same bound on the $H^{s}$-norm. 
Our main results in this paper are the following two theorems:

THEOREM 1. Assume that matrix (4) is positive definite. Initial value problem (1) is globally well-posed from data $\left(u_{10}, u_{20}\right) \in H^{s}\left(\mathbb{R}^{2}\right) \times H^{s}\left(\mathbb{R}^{2}\right)$ with $s>4 / 7$.

THEOREM 2. Assume that matrix (4) is positive definite. Initial value problem (1) is globally well-posed from data $\left(u_{10}, u_{20}\right) \in H^{s}\left(\mathbb{R}^{3}\right) \times H^{s}\left(\mathbb{R}^{3}\right)$ with $s>5 / 6$.

We remark that our assumption (4) is crucial to the globally well-posed solution to the system. Similar condition has been used in our blow-up result and stability result treated in our previous works [11] and [13]. Generally speaking, system (1) has more phenomenon than the scalar case to discover.

The rest of this paper is organized as follows: In Section 2, we introduce some notations and preliminaries. In Section 3, we will consider (1) and prove Theorem 1. In Section 4, we will deal with (1) and prove Theorem 2.

2. Preliminaries. Let us recall some well-known notations. Denote $\langle A\rangle \equiv(1+$ $\left.A^{2}\right)^{\frac{1}{2}}$ and $\left\langle\nabla^{F}\right\rangle$ for the operator with Fourier multiplier $\left(1+|\xi|^{2}\right)^{\frac{1}{2}}$, while the symbol $\nabla$ denotes the spatial gradient. Let $\frac{1}{2}+\equiv \frac{1}{2}+\varepsilon, \frac{1}{2}-\equiv \frac{1}{2}-\varepsilon$ and $\frac{1}{2}--\equiv \frac{1}{2}-2 \varepsilon$ for some universal $0<\varepsilon \ll 1$. Define the weighted Sobolev norms

$$
\begin{aligned}
\|\psi\|_{X_{s, b}} & \equiv\left\|\langle\xi\rangle^{s}\left\langle\tau-|\xi|^{2}\right\rangle^{b} \widehat{\psi}(\xi, \tau)\right\|_{L^{2}\left(\mathbb{R}^{n} \times \mathbb{R}\right)}, \\
\|\psi\|_{X_{s, b}^{\delta}} & \equiv \inf _{\psi=f \text { on }[0, \delta]}\|\psi\|_{X_{s, b}^{\delta}}, \\
\|F\|_{L_{t}^{q} L_{x}^{r}\left(\mathbb{R}^{n+1}\right)} & \equiv\left(\int_{\mathbb{R}}\left(\int_{\mathbb{R}^{n}}|F(x, t)|^{r} d x\right)^{\frac{q}{r}} d t\right)^{\frac{1}{q}} .
\end{aligned}
$$

A pair of exponents $(q, r)$ is called Schrödinger admissible for $R^{n+1}$ if $q$ and $r$ satisfy

$$
\frac{1}{q}+\frac{n}{2 r}=\frac{n}{4}, \quad q, r \geq 2, \quad(n, q) \neq(2,2) .
$$

For a Schrödinger admissible pair $(q, r)$ and a function $F(x, t)$ on $\mathbb{R}^{n+1}$, we have the $L_{t}^{q} L_{x}^{r}$ Strichartz estimate (see $\left.[3,15]\right)$

$$
\|F\|_{L_{t}^{q} L_{x}^{r}\left(\mathbb{R}^{n+1}\right)} \leq C\|F\|_{X_{0, \frac{1}{2}+}} .
$$

We next recall a lemma given by Bourgain [2] and Colliander [6].

LEMMA 2.1. Let $\psi_{1}, \psi_{2} \in X_{0, \frac{1}{2}+}^{\delta}$ be supported on spatial frequencies $|\xi| \sim N_{1}, N_{2}$, respectively. Then for any $N_{1} \leq N_{2}$

$$
\left\|\psi_{1} \cdot \psi_{2}\right\|_{L^{2}\left([0, \delta] \times \mathbb{R}^{2}\right)} \leq C\left(\frac{N_{1}}{N_{2}}\right)^{\frac{1}{2}}\left\|\psi_{1}\right\|_{X_{0, \frac{1}{2}+}^{\delta}}\left\|\psi_{2}\right\|_{X_{0, \frac{1}{2}+}^{\delta}} .
$$

holds. Moreover, if we replace the product $\psi_{1} \cdot \psi_{2}$ on the left with either $\bar{\psi}_{1} \cdot \psi_{2}$ or $\psi_{1} \cdot \bar{\psi}_{2}$, (15) also holds.

3. The case of $n=2$. As argued by Colliander et al. [6], to prove Theorem 1, it is sufficient to prove the following proposition: 
Proposition 3.1. Assume that $4 / 7<s$ and $u_{10}(x), u_{20}(x) \in C_{0}^{\infty}\left(\mathbb{R}^{2}\right)$ with $E\left(I u_{10}, I u_{20}\right) \leq 1$. Then there exists constant $\delta=\delta\left(\left\|u_{10}\right\|_{L^{2}\left(\mathbb{R}^{2}\right)},\left\|u_{20}\right\|_{L^{2}\left(\mathbb{R}^{2}\right)}\right)$ such that the solution $\left(u_{1}, u_{2}\right)$ satisfies

$$
u_{1}(x, t) \in C\left([0, \delta], H^{s}\left(\mathbb{R}^{2}\right)\right), \quad u_{2}(x, t) \in C\left([0, \delta], H^{s}\left(\mathbb{R}^{2}\right)\right)
$$

and

$$
E\left(I_{N} u_{1}, I_{N} u_{2}\right)(t)=E\left(I_{N} u_{1}, I_{N} u_{2}\right)(0)+O\left(N^{-\frac{3}{2}+}\right)
$$

for all $t \in[0, \delta]$.

In fact, Proposition 3.1 implies (9) in the following way. By scaling invariance, if $\left(u_{1}, u_{2}\right)$ is a solution to $(1)$, so does

$$
\left(u_{1}^{(\lambda)}(x, t), u_{2}^{(\lambda)}(x, t)\right):=\left(\frac{1}{\lambda} u_{1}\left(\frac{x}{\lambda}, \frac{t}{\lambda^{2}}\right), \frac{1}{\lambda} u_{2}\left(\frac{x}{\lambda}, \frac{t}{\lambda^{2}}\right)\right) .
$$

For all $\lambda>0$, we have the following estimate, which is similar to case of the single Schrödinger equation:

$$
\begin{aligned}
E\left(I_{N} u_{10}^{(\lambda)}, I_{N} u_{20}^{(\lambda)}\right)= & \frac{1}{2} \int_{\mathbb{R}^{2}}\left(\left|\nabla I_{N} u_{10}^{(\lambda)}\right|^{2}+\left|\nabla I_{N} u_{20}^{(\lambda)}\right|^{2}\right) d x \\
& +\frac{1}{4} \int_{\mathbb{R}^{2}}\left(\mu_{1}\left|I_{N} u_{10}^{(\lambda)}\right|^{4}+\mu_{2}\left|I_{N} u_{20}^{(\lambda)}\right|^{4}+2 \beta\left|I_{N} u_{10}^{(\lambda)}\right|^{2}\left|I_{N} u_{20}^{(\lambda)}\right|^{2}\right) d x \\
\leq & C\left(\lambda^{-2 s} N^{2-2 s}+\lambda^{-2}\right)\left(1+\left\|u_{10}\right\|_{H^{s}\left(\mathbb{R}^{2}\right)}+\left\|u_{20}\right\|_{H^{s}\left(\mathbb{R}^{2}\right)}\right)^{4} .
\end{aligned}
$$

Moreover, we can choose

$$
\lambda=\max \left\{\frac{1}{N}, \frac{N^{\frac{1-s}{s}}}{(2 C)^{\frac{1}{2 s}}}\left(1+\left\|u_{10}\right\|_{H^{s}\left(\mathbb{R}^{2}\right)}+\left\|u_{20}\right\|_{H^{s}\left(\mathbb{R}^{2}\right)}\right)^{\frac{2}{s}}\right\}
$$

to achieve $E\left(I_{N} u_{10}^{(\lambda)}, I_{N} u_{20}^{(\lambda)}\right) \leq \frac{1}{2}$. Reapplying Proposition 3.1 at least $C_{1} \cdot N^{\frac{3}{2}-}$ times, we arrive at

$$
E\left(I_{N} u_{1}^{(\lambda)}, I_{N} u_{2}^{(\lambda)}\right)\left(C_{1} \cdot N^{\frac{3}{2}-} \delta\right) \sim 1
$$

Choosing $N^{\frac{7 s-4}{2 s}-} \sim T_{0}$ and re-scaling back to the original energy, we obtain the desired bound

$$
E\left(I_{N} u_{1}, I_{N} u_{2}\right)(T) \leq C(1+T)^{\frac{4-4 s}{7-4 s}+} .
$$

For further details, refer to [6].

To prove Proposition 3.1, we need the following proposition:

PROPOSITION 3.2. Assume that $4 / 7<s<1$ and we are given the data for problem (1) with $E\left(I u_{10}, I u_{20}\right) \leq 1$. Then there exist constants $\delta=\delta\left(\left\|u_{10}\right\|_{L^{2}\left(\mathbb{R}^{2}\right)},\left\|u_{20}\right\|_{L^{2}\left(\mathbb{R}^{2}\right)}\right)$ and $C$ such that the solution $(u, v)$ satisfies

$$
\left\|I u_{1}\right\|_{X_{1, \frac{1}{2}+}^{\delta}}+\left\|I u_{2}\right\|_{X_{1, \frac{1}{2}+}^{\delta}} \ll C .
$$



$F(x, t)$

Proof. First we recall some estimates involving the $X_{s, b}^{\delta}$ spaces and functions $f(x)$,

$$
\begin{gathered}
\|S(t) f\|_{X_{1, \frac{1}{2}+}^{\delta}} \ll\|f\|_{H^{1}\left(\mathbb{R}^{n}\right)}, \\
\left\|\int_{0}^{t} S(t-\tau) F(x, \tau) d \tau\right\|_{X_{1, \frac{1}{2}+}} \ll\|F\|_{X_{1,-\frac{1}{2}}^{\delta}}, \\
\|F\|_{X_{1,-b}^{\delta}} \ll \delta^{P}\|F\|_{X_{1,-\alpha}^{\delta}},
\end{gathered}
$$

where $0<\alpha<b<\frac{1}{2}, P=\frac{1}{2}\left(1-\frac{\alpha}{b}\right)>0$.

Duhamel's principle gives us

$$
\begin{aligned}
&\left\|I u_{1}\right\|_{X_{1, \frac{1}{2}+}^{\delta}}=\left\|S(t)\left(I u_{10}\right)+\int_{0}^{t} S(t-\tau)\left(\mu_{1} I\left(u_{1} \bar{u}_{1} u_{1}\right)+\beta I\left(u_{2} \bar{u}_{2} u_{1}\right)\right)\right\|_{X_{1, \frac{1}{2}+}^{\delta}} \\
& \leq\left\|I u_{10}\right\|_{H^{1}\left(\mathbb{R}^{2}\right)}+\mu_{1}\left\|I\left(u_{1} \bar{u}_{1} u_{1}\right)\right\|_{X_{1,-\frac{1}{2}+}^{\delta}}+|\beta|\left\|I\left(u_{2} \bar{u}_{2} u_{1}\right)\right\|_{X_{1,-\frac{1}{2}+}^{\delta}} . \\
& \leq\left\|I u_{10}\right\|_{H^{1}\left(\mathbb{R}^{2}\right)}+\delta^{\varepsilon} \mu_{1}\left\|I\left(u_{1} \bar{u}_{1} u_{1}\right)\right\|_{X_{1,-\frac{1}{2}++}^{\delta}}+\delta^{\varepsilon}|\beta|\left\|I\left(u_{2} \bar{u}_{2} u_{1}\right)\right\|_{X_{1,-\frac{1}{2}++}^{\delta}} . \\
&\left\|I u_{2}\right\|_{X_{1, \frac{1}{2}+}^{\delta}} \leq\left\|I u_{20}\right\|_{H^{1}\left(\mathbb{R}^{2}\right)}+\delta^{\varepsilon} \mu_{2}\left\|I\left(u_{2} \bar{u}_{2} u_{2}\right)\right\|_{X_{1,-\frac{1}{2}++}^{\delta}}+\delta^{\varepsilon}|\beta|\left\|I\left(u_{1} \bar{u}_{1} u_{2}\right)\right\|_{X_{1,-\frac{1}{2}++}^{\delta}} .
\end{aligned}
$$

By the definition of the restricted norm, we have

$$
\begin{aligned}
& \left\|I u_{1}\right\|_{X_{1, \frac{1}{2}+}^{\delta}} \leq\left\|I u_{10}\right\|_{H^{1}\left(\mathbb{R}^{2}\right)}+\delta^{\varepsilon} \mu_{1}\left\|I\left(\psi_{1} \bar{\psi}_{1} \psi_{1}\right)\right\|_{X_{1,-\frac{1}{2}++}^{\delta}}+\delta^{\varepsilon}|\beta|\left\|I\left(\psi_{2} \bar{\psi}_{2} \psi_{1}\right)\right\|_{X_{1,-\frac{1}{2}++}^{\delta}}, \\
& \left\|I u_{2}\right\|_{X_{1, \frac{1}{2}+}^{\delta}} \leq\left\|I u_{20}\right\|_{H^{1}\left(\mathbb{R}^{2}\right)}+\delta^{\varepsilon} \mu_{2}\left\|I\left(\psi_{2} \bar{\psi}_{2} \psi_{2}\right)\right\|_{X_{1,-\frac{1}{2}++}^{\delta}}+\delta^{\varepsilon}|\beta|\left\|I\left(\psi_{1} \bar{\psi}_{1} \psi_{2}\right)\right\|_{X_{1,-\frac{1}{2}++}^{\delta}},
\end{aligned}
$$

where the function $\psi_{1}$ agrees with $u_{1}$ and $\psi_{2}$ agrees with $u_{2}$ for $t \in[0, \delta)$ and

$$
\left\|I u_{1}\right\|_{X_{1, \frac{1}{2}+}^{\delta}} \sim\left\|I \psi_{1}\right\|_{X_{1, \frac{1}{2}+}}, \quad\left\|I u_{2}\right\|_{X_{1, \frac{1}{2}+}^{\delta}} \sim\left\|I \psi_{2}\right\|_{X_{1, \frac{1}{2}+}} .
$$

By the results of [6], we know that

$$
\begin{aligned}
& \left\|I\left(\psi_{1} \bar{\psi}_{1} \psi_{1}\right)\right\|_{X_{1,-\frac{1}{2}++}} \ll\left\|I \psi_{1}\right\|_{X_{1, \frac{1}{2}+}}^{3}, \\
& \left\|I\left(\psi_{2} \bar{\psi}_{2} \psi_{2}\right)\right\|_{X_{1,-\frac{1}{2}++}} \ll\left\|I \psi_{2}\right\|_{X_{1, \frac{1}{2}+}}^{3} .
\end{aligned}
$$

Now we only need to show that

$$
\begin{aligned}
& \left\|I\left(\psi_{1} \bar{\psi}_{1} \psi_{2}\right)\right\|_{X_{1,-\frac{1}{2}++}} \ll\left\|I \psi_{1}\right\|_{X_{1, \frac{1}{2}+}}^{2}\left\|I \psi_{2}\right\|_{X_{1, \frac{1}{2}+}} \\
& \left\|I\left(\psi_{2} \bar{\psi}_{2} \psi_{1}\right)\right\|_{X_{1,-\frac{1}{2}++}} \ll\left\|I \psi_{2}\right\|_{X_{1, \frac{1}{2}+}}^{2}\left\|I \psi_{1}\right\|_{X_{1, \frac{1}{2}+}}
\end{aligned}
$$

We only prove (30), the proof of (31) is similar. Using the interpolation lemma of [7], we need to prove that

$$
\left\|\psi_{1} \bar{\psi}_{1} \psi_{2}\right\|_{X_{s,-\frac{1}{2}++}} \ll\left\|\psi_{1}\right\|_{X_{s, \frac{1}{2}+}}^{2}\left\|\psi_{2}\right\|_{X_{s, \frac{1}{2}+}}
$$


for all $4 / 7<s<1$. However, by duality and 'Leibniz' rule (32) follows from

$$
\left|\int_{\mathbb{R}} \int_{\mathbb{R}^{2}}\left(\left\langle\nabla^{F}\right\rangle^{s} \phi_{1}\right) \bar{\phi}_{2} \phi_{3} \phi_{4} d x d t\right| \ll\left\|\phi_{1}\right\|_{X_{s, \frac{1}{2}+}}\left\|\phi_{2}\right\|_{X_{s, \frac{1}{2}+}}\left\|\phi_{3}\right\|_{X_{s, \frac{1}{2}+}}\left\|\phi_{4}\right\|_{X_{0, \frac{1}{2}--}} .
$$

Hence (30) is obtained.

Setting

$$
Q_{1}(\delta)=\left\|I u_{1}\right\|_{X_{1, \frac{1}{2}+}^{\delta}}, \quad Q_{2}(\delta)=\left\|I u_{2}\right\|_{X_{1, \frac{1}{2}+}^{\delta}}
$$

we have

$$
\begin{aligned}
& Q_{1}(\delta) \leq\left\|I u_{10}\right\|_{H^{1}\left(\mathbb{R}^{2}\right)}+\delta^{\varepsilon} \mu_{1}\left(Q_{1}(\delta)\right)^{3}+\delta^{\varepsilon}|\beta|\left(Q_{2}(\delta)\right)^{2} Q_{1}(\delta), \\
& Q_{2}(\delta) \leq\left\|I u_{20}\right\|_{H^{1}\left(\mathbb{R}^{2}\right)}+\delta^{\varepsilon} \mu_{2}\left(Q_{2}(\delta)\right)^{3}+\delta^{\varepsilon}|\beta|\left(Q_{1}(\delta)\right)^{2} Q_{2}(\delta) .
\end{aligned}
$$

Summing them up, we can obtain that

$$
Q_{1}(\delta)+Q_{2}(\delta) \leq\left\|I u_{10}\right\|_{H^{1}\left(\mathbb{R}^{2}\right)}+\left\|I u_{20}\right\|_{H^{1}\left(\mathbb{R}^{2}\right)}+C \delta^{\varepsilon}\left(Q_{1}(\delta)+Q_{2}(\delta)\right)^{3} .
$$

We emphasize that the above inequality is the analogue of the inequality (3.23) in [6] related to the single Schrödinger equation. The framework of [6] yields the desired result in Proposition 3.2.

Now we give the proof of Proposition 3.1.

Proof of Proposition 3.1. Applying I to equation (1), we can obtain that

$$
\begin{aligned}
\partial_{t} E\left(I u_{1}, I u_{2}\right)= & \operatorname{Re} \int_{\mathbb{R}^{2}}\left\{\overline{I u}_{1 t}\left(\mu_{1}\left|I u_{1}\right|^{2} I u_{1}+\beta\left|I u_{2}\right|^{2} I u_{1}-I\left(\mu_{1}\left|u_{1}\right|^{2} u_{1}+\beta\left|u_{2}\right|^{2} u_{1}\right)\right)\right. \\
& \left.+\overline{I u}_{2 t}\left(\mu_{2}\left|I u_{2}\right|^{2} I u_{2}+\beta\left|I u_{1}\right|^{2} I u_{2}-I\left(\mu_{2}\left|u_{2}\right|^{2} u_{2}+\beta\left|u_{1}\right|^{2} u_{2}\right)\right)\right\} d x
\end{aligned}
$$

Integrating (35) from 0 to $\delta$, we have

$$
\begin{aligned}
& E\left(I u_{1}, I u_{2}\right)(\delta)-E\left(I u_{1}, I u_{2}\right)(0) \\
& =\int_{0}^{\delta} \int_{\sum_{j=1}^{4} \zeta_{j}=0}\left(1-\frac{M\left(\zeta_{2}+\zeta_{3}+\zeta_{4}\right)}{M\left(\zeta_{2}\right) M\left(\zeta_{3}\right) M\left(\zeta_{4}\right)}\right) \widehat{\mu_{1}} \widehat{\overline{I \partial_{t} u_{1}}}\left(\zeta_{1}\right) \widehat{I u_{1}}\left(\zeta_{2}\right) \widehat{I u_{1}}\left(\zeta_{3}\right) \widehat{I u_{1}}\left(\zeta_{4}\right) \\
& +\int_{0}^{\delta} \int_{\sum_{j=1}^{4} \zeta_{j}=0}\left(1-\frac{M\left(\zeta_{2}+\zeta_{3}+\zeta_{4}\right)}{M\left(\zeta_{2}\right) M\left(\zeta_{3}\right) M\left(\zeta_{4}\right)}\right) \widehat{\beta \overline{I \partial_{t} u_{1}}}\left(\zeta_{1}\right) \widehat{I u_{2}}\left(\zeta_{2}\right) \widehat{I u_{2}}\left(\zeta_{3}\right) \widehat{I u_{1}}\left(\zeta_{4}\right) \\
& +\int_{0}^{\delta} \int_{\sum_{j=1}^{4} \eta_{j}=0}\left(1-\frac{M\left(\eta_{2}+\eta_{3}+\eta_{4}\right)}{M\left(\eta_{2}\right) M\left(\eta_{3}\right) M\left(\eta_{4}\right)}\right) \widehat{\mu_{2}} \widehat{\overline{I \partial_{t} u_{2}}}\left(\eta_{1}\right) \widehat{I u_{2}}\left(\eta_{2}\right) \widehat{\overline{I u_{2}}}\left(\eta_{3}\right) \widehat{I u_{2}}\left(\eta_{4}\right) \\
& +\int_{0}^{\delta} \int_{\sum_{j=1}^{4} \eta_{j}=0}\left(1-\frac{M\left(\eta_{2}+\eta_{3}+\eta_{4}\right)}{M\left(\eta_{2}\right) M\left(\eta_{3}\right) M\left(\eta_{4}\right)}\right) \widehat{\beta \overline{I \partial_{t} u_{2}}}\left(\eta_{1}\right) \widehat{I u_{1}}\left(\eta_{2}\right) \widehat{\overline{I u_{1}}}\left(\eta_{3}\right) \widehat{I u_{2}}\left(\eta_{4}\right) \text {. }
\end{aligned}
$$

Denote

$$
\begin{aligned}
& L_{1}(\zeta)=\left(1-\frac{M\left(\zeta_{2}+\zeta_{3}+\zeta_{4}\right)}{M\left(\zeta_{2}\right) M\left(\zeta_{3}\right) M\left(\zeta_{4}\right)}\right) \\
& L_{2}(\eta)=\left(1-\frac{M\left(\eta_{2}+\eta_{3}+\eta_{4}\right)}{M\left(\eta_{2}\right) M\left(\eta_{3}\right) M\left(\eta_{4}\right)}\right)
\end{aligned}
$$


Using the equations of (1), we substitute for $\partial_{t} u_{1}$ and $\partial_{t} u_{2}$ in (36) and we will show that

$$
\sum_{j=1}^{8} \operatorname{Term}_{j} \ll N^{-\frac{3}{2}+},
$$

where

$$
\begin{aligned}
& \operatorname{Term}_{1}=\int_{0}^{\delta} \int_{\sum_{j=1}^{4} \zeta_{j}=0} \mu_{1} L_{1}(\zeta) \widehat{\overline{\Delta I u_{1}}}\left(\zeta_{1}\right) \widehat{I u_{1}}\left(\zeta_{2}\right) \widehat{\overline{I u_{1}}}\left(\zeta_{3}\right) \widehat{I u_{1}}\left(\zeta_{4}\right)
\end{aligned}
$$

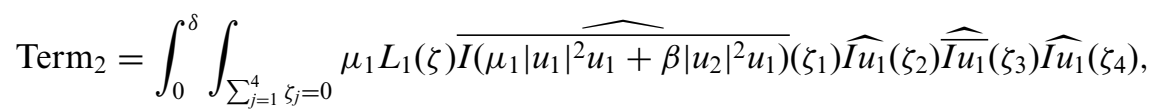

$$
\begin{aligned}
& \operatorname{Term}_{3}=\int_{0}^{\delta} \int_{\sum_{j=1}^{4} \zeta_{j}=0} \beta L_{1}(\zeta) \widehat{\widehat{\Delta I u_{1}}}\left(\zeta_{1}\right) \widehat{\mathscr{I u _ { 2 }}}\left(\zeta_{2}\right) \widehat{\overline{I u_{2}}}\left(\zeta_{3}\right) \widehat{I u_{1}}\left(\zeta_{4}\right), \\
& \operatorname{Term}_{4}=\int_{0}^{\delta} \int_{\sum_{j=1}^{4} \zeta_{j}=0} \beta L_{1}(\zeta) \overline{I\left(\mu_{1}\left|u_{1}\right|^{2} u_{1}+\beta\left|u_{2}\right|^{2} u_{1}\right)}\left(\zeta_{1}\right) \widehat{I u_{2}}\left(\zeta_{2}\right) \widehat{\overline{I u_{2}}}\left(\zeta_{3}\right) \widehat{I u_{1}}\left(\zeta_{4}\right), \\
& \operatorname{Term}_{5}=\int_{0}^{\delta} \int_{\sum_{j=1}^{4} \zeta_{j}=0} \mu_{2} L_{2}(\eta) \widehat{\overline{\Delta I u_{2}}}\left(\eta_{1}\right) \widehat{I u_{2}}\left(\eta_{2}\right) \widehat{\overline{I u_{2}}}\left(\eta_{3}\right) \widehat{I u_{2}}\left(\eta_{4}\right), \\
& \operatorname{Term}_{6}=\int_{0}^{\delta} \int_{\sum_{j=1}^{4} \zeta_{j}=0} \mu_{2} L_{2}(\eta) \overline{I\left(\mu_{2}\left|u_{2}\right|^{2} u_{2}+\beta\left|u_{1}\right|^{2} u_{2}\right)}\left(\eta_{1}\right) \widehat{I u_{2}}\left(\eta_{2}\right) \widehat{\overline{I u_{2}}}\left(\eta_{3}\right) \widehat{I u_{2}}\left(\eta_{4}\right), \\
& \operatorname{Term}_{7}=\int_{0}^{\delta} \int_{\sum_{j=1}^{4} \zeta_{j}=0} \beta L_{2}(\eta) \widehat{\widehat{\Delta I u_{2}}}\left(\eta_{1}\right) \widehat{I u_{1}}\left(\eta_{2}\right) \widehat{\overline{I u_{1}}}\left(\eta_{3}\right) \widehat{I u_{2}}\left(\eta_{4}\right), \\
& \operatorname{Term}_{8}=\int_{0}^{\delta} \int_{\sum_{j=1}^{4} \zeta_{j}=0} \beta L_{2}(\eta) \widehat{I\left(\mu_{2}\left|u_{2}\right|^{2} u_{2}+\beta\left|u_{1}\right|^{2} u_{2}\right)}\left(\eta_{1}\right) \widehat{I u_{1}}\left(\eta_{2}\right) \widehat{\overline{I u_{1}}}\left(\eta_{3}\right) \widehat{I u_{2}}\left(\eta_{4}\right) .
\end{aligned}
$$

By the results of [6], we know that

$$
\begin{aligned}
& \left|\int_{0}^{\delta} \int_{\sum_{j=1}^{4} \zeta_{j}=0}\left(1-\frac{M\left(\zeta_{2}+\zeta_{3}+\zeta_{4}\right)}{M\left(\zeta_{2}\right) M\left(\zeta_{3}\right) M\left(\zeta_{4}\right)}\right) \widehat{\bar{\phi}_{1}}\left(\zeta_{1}\right) \widehat{\phi_{2}}\left(\zeta_{2}\right) \widehat{\bar{\phi}_{3}}\left(\zeta_{3}\right) \widehat{\phi_{4}}\left(\zeta_{4}\right)\right| \\
& \leq C N^{-\frac{3}{2}}\left(N_{1} N_{2} N_{3} N_{4}\right)^{0-}\left\|\phi_{1}\right\|_{X_{-1, \frac{1}{2}+}}\left\|\phi_{2}\right\|_{X_{1, \frac{1}{2}+}}\left\|\phi_{3}\right\|_{X_{1, \frac{1}{2}+}}\left\|\phi_{4}\right\|_{X_{1, \frac{1}{2}+}}
\end{aligned}
$$

for any functions $\phi_{j}(j=1, \ldots, 4)$ with positive spatial Fourier transforms supported on

$$
\langle\xi\rangle \sim 2^{k_{j}} \equiv N_{j}
$$

for some $k_{j} \in\{0,1, \ldots\}$. that

Letting $\phi_{1}=\Delta I u_{k}(k=1,2), \phi_{j}=I u_{k}(j=2,3,4, k=1,2)$ in (46), we obtain

$$
\operatorname{Term}_{1}+\operatorname{Term}_{3}+\operatorname{Term}_{5}+\operatorname{Term}_{7} \leq C N^{-\frac{3}{2}} .
$$

Now we need to estimate Term 2, Term $_{4}$, Term $_{6}$, Term $_{8}$.

First we consider Term 2 . 
It is easy to see that $\mid$ Term $_{2}|\leq|$ Term $_{21}|+|$ Term $_{22} \mid$ with

$$
\begin{aligned}
\operatorname{Term}_{21} & =\int_{0}^{\delta} \int_{\sum_{j=1}^{4} \zeta_{j}=0} \mu_{1} L_{1}(\zeta) \overline{I\left(\mu_{1}\left|u_{1}\right|^{2} u_{1}\right)}\left(\zeta_{1}\right) \widehat{I u_{1}}\left(\zeta_{2}\right) \widehat{I u_{1}}\left(\zeta_{3}\right) \widehat{I u_{1}}\left(\zeta_{4}\right), \\
\operatorname{Term}_{22} & =\int_{0}^{\delta} \int_{\sum_{j=1}^{4} \zeta_{j}=0} \mu_{1} L_{1}(\zeta) \widehat{I\left(\beta\left|u_{2}\right|^{2} u_{1}\right)}\left(\zeta_{1}\right) \widehat{I u_{1}}\left(\zeta_{2}\right) \widehat{I u_{1}}\left(\zeta_{3}\right) \widehat{I u_{1}}\left(\zeta_{4}\right)
\end{aligned}
$$

By the results of [6], we know that

$$
\begin{aligned}
& \left|\int_{0}^{\delta} \int_{\sum_{j=1}^{6} \zeta_{j}=0} L_{1}(\zeta) P_{N_{123}} \overline{I\left(\phi_{1} \phi_{2} \phi_{3}\right)}\left(\zeta_{1}+\zeta_{2}+\zeta_{3}\right) \widehat{I \phi_{4}}\left(\zeta_{4}\right) \widehat{\overline{I\left(\phi_{5}\right)}}\left(\zeta_{5}\right) \widehat{I \phi_{6}}\left(\zeta_{6}\right)\right| \\
& \leq C N^{-\frac{3}{2}} N_{4}^{0-} \prod_{j=1}^{6}\left\|I \phi_{j}\right\|_{X_{1, \frac{1}{2}+}}
\end{aligned}
$$

where $0 \leq \hat{\phi}_{j}$ is supported for $\left|\xi_{j}\right| \sim N_{j}=2^{k_{j}}$ and

$$
N_{4} \geq N_{5} \geq N_{6} \quad \text { and } N_{4} \geq C N,
$$

while let $P_{N_{123}}$ be the projection onto functions supported in the $N_{123}$ dyadic spatial frequency shell.

Letting $\phi_{j}=u_{1}(j=1,2, \ldots, 6)$ in Term 21 and $\phi_{1}=\phi_{2}=u_{2}, \phi_{j}=u_{1}(j=3,4,5$, 6) in Term 22 , using (49), and proceeding exactly as in [6], we finally obtain that

$$
\left|\operatorname{Term}_{2}\right| \leq C N^{-\frac{3}{2}}
$$

Similarly, we can get the bounds for $\mathrm{Term}_{4}, \mathrm{Term}_{6}, \mathrm{Term}_{8}$. Proposition 3.1 is proved.

4. The results in $\mathbb{R}^{3}$. Similar to the proof of Theorem 1 , we only need to prove the following proposition:

Proposition 4.1. Assume that $s>5 / 6, N \gg 1,\left(u_{10}, u_{20}\right) \in C_{0}^{\infty}\left(\mathbb{R}^{3}\right) \times C_{0}^{\infty}\left(\mathbb{R}^{3}\right)$ with $E\left(I_{N} u_{10}, I_{N} u_{20}\right) \leq 1$. Then there exists a universal constant $\delta$ such that

$$
(u, v) \in C\left([0, \delta], H^{s}\left(\mathbb{R}^{3}\right)\right) \times C\left([0, \delta], H^{s}\left(\mathbb{R}^{3}\right)\right)
$$

satisfies

$$
E\left(I_{N} u_{1}, I_{N} u_{2}\right)(t)=E\left(I_{N} u_{1}, I_{N} u_{2}\right)(0)+O\left(N^{-1+}\right)
$$

for all $t \in[0, \delta]$.

Recall too $\left(u_{1}^{(\lambda)}(x, t), u_{2}^{(\lambda)}(x, t)\right)$ as the scaled solution defined in (17). For $n=3$ and all $\lambda>0$, we have

$$
\begin{aligned}
E\left(I_{N} u_{10}^{(\lambda)}, I_{N} u_{20}^{(\lambda)}\right) & =\frac{1}{2} \int_{\mathbb{R}^{3}}\left(\left|\nabla I_{N} u_{10}^{(\lambda)}\right|^{2}+\left|\nabla I_{N} u_{20}^{(\lambda)}\right|^{2}\right) d x \\
& \leq C_{0} \lambda^{1-2 s} N^{2-2 s}\left(1+\left\|u_{10}\right\|_{H^{s}\left(\mathbb{R}^{3}\right)}+\left\|u_{20}\right\|_{H^{s}\left(\mathbb{R}^{3}\right)}\right)^{4} .
\end{aligned}
$$


Moreover, we can choose

$$
\left.\lambda=\frac{N^{\frac{2 s-2}{1-2 s}}}{\left(2 C_{0}\right)^{\frac{1}{1-2 s}}}\left(1+\left\|u_{10}\right\|_{H^{s}\left(\mathbb{R}^{3}\right)}+\left\|u_{20}\right\|_{H^{s}\left(\mathbb{R}^{3}\right)}\right)^{-\frac{4}{1-2 s}}\right\}
$$

such that $E\left(I_{N} u_{10}^{(\lambda)}, I_{N} u_{20}^{(\lambda)}\right) \leq 1 / 2$. Reapplying Proposition 4.1 at least $C_{1} \times N^{1-}$ times, we get

$$
E\left(I_{N} u_{1}^{(\lambda)}, I_{N} u_{2}^{(\lambda)}\right)\left(C_{1} N^{1-} \times \delta\right) \sim 1
$$

Choosing $N^{\left(\frac{5-6 s}{1-2 s}\right)-} \sim T_{0}$ and re-scaling back to the original energy, and noticing that in three dimensions

$$
E\left(I_{N} u_{1}^{(\lambda)}, I_{N} u_{2}^{(\lambda)}\right)\left(\lambda^{2} t\right)=\frac{1}{\lambda} E\left(I_{N} u_{1}^{(\lambda)}, I_{N} u_{2}^{(\lambda)}\right)(t),
$$

we can obtain that

$$
E\left(I_{N} u_{1}^{(\lambda)}, I_{N} u_{2}^{(\lambda)}\right)\left(T_{0}\right) \leq \lambda E\left(I_{N} u_{1}^{(\lambda)}, I_{N} u_{2}^{(\lambda)}\right)\left(\lambda^{2} T_{0}\right) \leq C T_{0}^{\frac{1-s_{+}}{3(s-5 / 6)}} .
$$

Hence we can achieve that

$$
\left\|u_{1}\right\|_{H^{s}\left(\mathbb{R}^{3}\right)}(T)+\left\|u_{2}\right\|_{H^{s}\left(\mathbb{R}^{3}\right)}(T) \leq C(1+T)^{\frac{1-s_{+}}{6(s-5 / 6)}} .
$$

To prove Proposition 4.1, we need the following proposition:

Proposition 4.2. Assume that $5 / 6<s<1$ and $\left(u_{10}, u_{20}\right)$ satisfies $E\left(u_{10}, u_{20}\right) \leq 1$. Then there exist a universal constant $\delta>0$ such that

$$
\left\|\nabla u_{1}\right\|_{X_{0, \frac{1}{2}+}^{\delta}}+\left\|\nabla u_{2}\right\|_{X_{0, \frac{1}{2}+}^{\delta}} \leq C
$$

Proof. Using Duhamel's principle, we get that

$$
\begin{aligned}
\left\|\nabla I u_{1}\right\|_{X_{1, \frac{1}{2}+}^{\delta} \leq} & \left\|\nabla I u_{10}\right\|_{H^{1}\left(\mathbb{R}^{2}\right)}+\delta^{\varepsilon} \mu_{1}\left\|\nabla I\left(u_{1} \bar{u}_{1} u_{1}\right)\right\|_{X_{1,-\frac{1}{2}++}^{\delta}} \\
& +\delta^{\varepsilon}|\beta|\left\|\nabla I\left(u_{2} \bar{u}_{2} u_{1}\right)\right\|_{X_{1,-\frac{1}{2}++}^{\delta}}, \\
\left\|\nabla I u_{2}\right\|_{X_{1, \frac{1}{2}+}^{\delta} \leq} \leq & \left\|\nabla I u_{20}\right\|_{H^{1}\left(\mathbb{R}^{2}\right)}+\delta^{\varepsilon} \mu_{2}\left\|\nabla I\left(u_{2} \bar{u}_{2} u_{2}\right)\right\|_{X_{1,-\frac{1}{2}++}^{\delta}} \\
& +\delta^{\varepsilon}|\beta|\left\|\nabla I\left(u_{1} \bar{u}_{1} u_{2}\right)\right\|_{X_{1,-\frac{1}{2}++}^{\delta}} .
\end{aligned}
$$

By the definition of the restricted norm, we have

$$
\begin{aligned}
\left\|\nabla I u_{1}\right\|_{X_{1, \frac{1}{2}+}^{\delta} \leq} \leq & \left\|\nabla I u_{10}\right\|_{H^{1}\left(\mathbb{R}^{2}\right)}+\delta^{\varepsilon} \mu_{1}\left\|\nabla I\left(\psi_{1} \bar{\psi}_{1} \psi_{1}\right)\right\|_{X_{1,-\frac{1}{2}++}^{\delta}} \\
& +\delta^{\varepsilon}|\beta|\left\|\nabla I\left(\psi_{2} \bar{\psi}_{2} \psi_{1}\right)\right\|_{X_{1,-\frac{1}{2}++}^{\delta}}, \\
\left\|\nabla I u_{2}\right\|_{X_{1, \frac{1}{2}+}^{\delta} \leq} \leq & \left\|\nabla I u_{20}\right\|_{H^{1}\left(\mathbb{R}^{2}\right)}+\delta^{\varepsilon} \mu_{2}\left\|\nabla I\left(\psi_{2} \bar{\psi}_{2} \psi_{2}\right)\right\|_{X_{1,-\frac{1}{2}++}^{\delta}} \\
& +\delta^{\varepsilon}|\beta|\left\|\nabla I\left(\psi_{1} \bar{\psi}_{1} \psi_{2}\right)\right\|_{X_{1,-\frac{1}{2}++}^{\delta}},
\end{aligned}
$$


where the function $\psi_{1}$ agrees with $u_{1}$ and $\psi_{2}$ agrees with $u_{2}$ for $t \in[0, \delta)$ and

$$
\left\|\nabla I u_{1}\right\|_{X_{1, \frac{1}{2}+}^{\delta}} \sim\left\|\nabla I \psi_{1}\right\|_{X_{1, \frac{1}{2}+}}, \quad\left\|\nabla I u_{2}\right\|_{X_{1, \frac{1}{2}+}^{\delta}} \sim\left\|\nabla I \psi_{2}\right\|_{X_{1, \frac{1}{2}+}} .
$$

By the results of [6], we know that

$$
\begin{aligned}
& \left\|\nabla I\left(u_{1} \bar{u}_{1} u_{1}\right)\right\|_{X_{0,-\frac{1}{2}++}^{\delta}} \ll\left\|\nabla I u_{1}\right\|_{X_{0, \frac{1}{2}+}}^{3}, \\
& \left\|\nabla I\left(u_{2} \bar{u}_{2} u_{2}\right)\right\|_{X_{0,-\frac{1}{2}++}^{\delta}} \ll\left\|\nabla I u_{2}\right\|_{X_{0, \frac{1}{2}+}^{3}}^{3} .
\end{aligned}
$$

Now we only need to prove that

$$
\begin{aligned}
& \left\|\nabla I\left(u_{1} \bar{u}_{1} u_{2}\right)\right\|_{X_{0,-\frac{1}{2}++}^{\delta}} \ll\left\|\nabla I u_{1}\right\|_{X_{0, \frac{1}{2}+}}^{2}\left\|\nabla I u_{2}\right\|_{X_{0, \frac{1}{2}+}}, \\
& \left\|\nabla I\left(u_{2} \bar{u}_{2} u_{1}\right)\right\|_{X_{0,-\frac{1}{2}++}^{\delta}} \ll\left\|\nabla I u_{2}\right\|_{X_{0, \frac{1}{2}+}}^{2}\left\|\nabla I u_{1}\right\|_{X_{0, \frac{1}{2}+}} .
\end{aligned}
$$

By the results of [6], and applying a Leibnitz rule for the operator $\nabla I$ and duality, one can show that

$$
\left\|(\nabla I)\left(\phi_{1}\right) \cdot \bar{\phi}_{2} \cdot \phi_{3} \cdot \psi\right\|_{L^{1}\left(\mathbb{R}^{3+1}\right)} \leq C\|\psi\|_{X_{0, \frac{1}{2}--}} \prod_{j=1}^{3}\left\|\nabla \phi_{j}\right\|_{X_{0, \frac{1}{2}+}} .
$$

Letting $\phi_{j}=u_{k}(j=1,2,3, k=1,2)$ in (67) and proceeding as in [6], we can obtain (65) and (66).

Setting

$$
Q_{1}(\delta)=\left\|\nabla I u_{1}\right\|_{X_{1, \frac{1}{2}+}^{\delta}}, \quad Q_{2}(\delta)=\left\|\nabla I u_{2}\right\|_{X_{1, \frac{1}{2}+}^{\delta}},
$$

we have

$$
\begin{aligned}
& Q_{1}(\delta) \leq\left\|\nabla I u_{10}\right\|_{H^{1}\left(\mathbb{R}^{2}\right)}+\delta^{\varepsilon} \mu_{1}\left(Q_{1}(\delta)\right)^{3}+\delta^{\varepsilon}|\beta|\left(Q_{2}(\delta)\right)^{2} Q_{1}(\delta), \\
& Q_{2}(\delta) \leq\left\|\nabla I u_{20}\right\|_{H^{1}\left(\mathbb{R}^{2}\right)}+\delta^{\varepsilon} \mu_{2}\left(Q_{2}(\delta)\right)^{3}+\delta^{\varepsilon}|\beta|\left(Q_{1}(\delta)\right)^{2} Q_{2}(\delta) .
\end{aligned}
$$

Summing them up, we obtain

$$
Q_{1}(\delta)+Q_{2}(\delta) \leq\left\|\nabla I u_{10}\right\|_{H^{1}\left(\mathbb{R}^{2}\right)}+\left\|\nabla I u_{20}\right\|_{H^{1}\left(\mathbb{R}^{2}\right)}+C \delta^{\varepsilon}\left(Q_{1}(\delta)+Q_{2}(\delta)\right)^{3} .
$$

And by continuity we obtain

$$
\left\|\nabla I u_{1}\right\|_{X_{1, \frac{1}{2}+}^{\delta}}+\left\|\nabla I u_{2}\right\|_{X_{1, \frac{1}{2}+}^{\delta}} \leq C .
$$

Proof of Proposition 4.1. Applying I to equation (1), we can obtain

$$
\begin{aligned}
\partial_{t} E\left(I u_{1}, I u_{2}\right)= & \operatorname{Re} \int_{\mathbb{R}^{3}}\left\{\overline{I u}_{1 t}\left(\mu_{1}\left|I u_{1}\right|^{2} I u_{1}+\beta\left|I u_{2}\right|^{2} I u_{1}-I\left(\mu_{1}\left|u_{1}\right|^{2} u_{1}+\beta\left|u_{2}\right|^{2} u_{1}\right)\right)\right. \\
& \left.+\overline{I u}_{2 t}\left(\mu_{2}\left|I u_{2}\right|^{2} I u_{2}+\beta\left|I u_{1}\right|^{2} I u_{2}-I\left(\mu_{2}\left|u_{2}\right|^{2} u_{2}+\beta\left|u_{1}\right|^{2} u_{2}\right)\right)\right\} d x
\end{aligned}
$$


Integrating (69) from 0 to $\delta$, we have

$$
\begin{aligned}
& E\left(I u_{1}, I u_{2}\right)(\delta)-E\left(I u_{1}, I u_{2}\right)(0) \\
& =\int_{0}^{\delta} \int_{\sum_{j=1}^{4} \zeta_{j}=0}\left(1-\frac{M\left(\zeta_{2}+\zeta_{3}+\zeta_{4}\right)}{M\left(\zeta_{2}\right) M\left(\zeta_{3}\right) M\left(\zeta_{4}\right)}\right) \widehat{\mu_{1}} \widehat{\overline{I \partial_{t} u_{1}}}\left(\zeta_{1}\right) \widehat{I u_{1}}\left(\zeta_{2}\right) \widehat{\overline{I u_{1}}}\left(\zeta_{3}\right) \widehat{I u_{1}}\left(\zeta_{4}\right) \\
& +\int_{0}^{\delta} \int_{\sum_{j=1}^{4} \zeta_{j}=0}\left(1-\frac{M\left(\zeta_{2}+\zeta_{3}+\zeta_{4}\right)}{M\left(\zeta_{2}\right) M\left(\zeta_{3}\right) M\left(\zeta_{4}\right)}\right) \widehat{\beta \overline{I \partial_{t} u_{1}}}\left(\zeta_{1}\right) \widehat{I u_{2}}\left(\zeta_{2}\right) \widehat{\overline{I u_{2}}}\left(\zeta_{3}\right) \widehat{I u_{1}}\left(\zeta_{4}\right) \\
& +\int_{0}^{\delta} \int_{\sum_{j=1}^{4} \eta_{j}=0}\left(1-\frac{M\left(\eta_{2}+\eta_{3}+\eta_{4}\right)}{M\left(\eta_{2}\right) M\left(\eta_{3}\right) M\left(\eta_{4}\right)}\right) \widehat{\overline{I \partial_{t} u_{2}}}\left(\eta_{1}\right) \widehat{I u_{2}}\left(\eta_{2}\right) \widehat{\overline{I u_{2}}}\left(\eta_{3}\right) \widehat{I u_{2}}\left(\eta_{4}\right) \\
& +\int_{0}^{\delta} \int_{\sum_{j=1}^{4} \eta_{j}=0}\left(1-\frac{M\left(\eta_{2}+\eta_{3}+\eta_{4}\right)}{M\left(\eta_{2}\right) M\left(\eta_{3}\right) M\left(\eta_{4}\right)}\right) \widehat{\beta \overline{I \partial_{t} u_{2}}}\left(\eta_{1}\right) \widehat{I u_{1}}\left(\eta_{2}\right) \widehat{\overline{I u_{1}}}\left(\eta_{3}\right) \widehat{I u_{2}}\left(\eta_{4}\right) \text {. }
\end{aligned}
$$

Denote

$$
\begin{aligned}
& L_{1}(\zeta)=\left(1-\frac{M\left(\zeta_{2}+\zeta_{3}+\zeta_{4}\right)}{M\left(\zeta_{2}\right) M\left(\zeta_{3}\right) M\left(\zeta_{4}\right)}\right), \\
& L_{2}(\eta)=\left(1-\frac{M\left(\eta_{2}+\eta_{3}+\eta_{4}\right)}{M\left(\eta_{2}\right) M\left(\eta_{3}\right) M\left(\eta_{4}\right)}\right) .
\end{aligned}
$$

Using the equations given in (1), we substitute for $\partial_{t} u_{1}$ and $\partial_{t} u_{2}$ in (70) and we will show that

$$
\sum_{j=1}^{8} \operatorname{Term}_{j} \ll N^{-1++}
$$

where

$$
\begin{aligned}
& \operatorname{Term}_{1}=\int_{0}^{\delta} \int_{\sum_{j=1}^{4} \zeta_{j}=0} \mu_{1} L_{1}(\zeta) \widehat{\widehat{\Delta I u_{1}}}\left(\zeta_{1}\right) \widehat{I u_{1}}\left(\zeta_{2}\right) \widehat{\overline{I u_{1}}}\left(\zeta_{3}\right) \widehat{I u_{1}}\left(\zeta_{4}\right)
\end{aligned}
$$

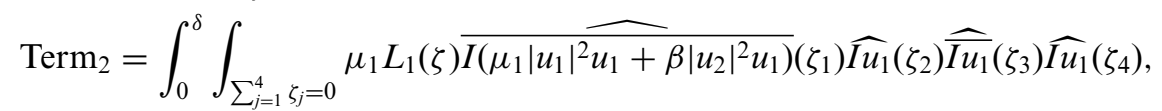

$$
\begin{aligned}
& \operatorname{Term}_{3}=\int_{0}^{\delta} \int_{\sum_{j=1}^{4} \zeta_{j}=0} \beta L_{1}(\zeta) \widehat{\overline{\Delta I u_{1}}}\left(\zeta_{1}\right) \widehat{I u_{2}}\left(\zeta_{2}\right) \widehat{\overline{I u_{2}}}\left(\zeta_{3}\right) \widehat{I u_{1}}\left(\zeta_{4}\right), \\
& \operatorname{Term}_{4}=\int_{0}^{\delta} \int_{\sum_{j=1}^{4} \zeta_{j}=0} \beta L_{1}(\zeta) \widehat{I\left(\mu_{1}\left|u_{1}\right|^{2} u_{1}+\beta\left|u_{2}\right|^{2} u_{1}\right)}\left(\zeta_{1}\right) \widehat{I u_{2}}\left(\zeta_{2}\right) \widehat{\overline{I u_{2}}}\left(\zeta_{3}\right) \widehat{I u_{1}}\left(\zeta_{4}\right), \\
& \operatorname{Term}_{5}=\int_{0}^{\delta} \int_{\sum_{j=1}^{4} \zeta_{j}=0} \mu_{2} L_{2}(\eta) \widehat{\overline{\Delta I u_{2}}}\left(\eta_{1}\right) \widehat{I u_{2}}\left(\eta_{2}\right) \widehat{\overline{I u_{2}}}\left(\eta_{3}\right) \widehat{I u_{2}}\left(\eta_{4}\right) \\
& \operatorname{Term}_{6}=\int_{0}^{\delta} \int_{\sum_{j=1}^{4} \zeta_{j}=0} \mu_{2} L_{2}(\eta) \overline{I\left(\mu_{2}\left|u_{2}\right|^{2} u_{2}+\beta\left|u_{1}\right|^{2} u_{2}\right)}\left(\eta_{1}\right) \widehat{I u_{2}}\left(\eta_{2}\right) \widehat{\overline{I u_{2}}}\left(\eta_{3}\right) \widehat{I u_{2}}\left(\eta_{4}\right) \text {, } \\
& \operatorname{Term}_{7}=\int_{0}^{\delta} \int_{\sum_{j=1}^{4} \zeta_{j}=0} \beta L_{2}(\eta) \widehat{\widehat{\Delta I u_{2}}}\left(\eta_{1}\right) \widehat{I u_{1}}\left(\eta_{2}\right) \widehat{\overline{I u_{1}}}\left(\eta_{3}\right) \widehat{I u_{2}}\left(\eta_{4}\right), \\
& \operatorname{Term}_{8}=\int_{0}^{\delta} \int_{\sum_{j=1}^{4} \zeta_{j}=0} \beta L_{2}(\eta) \widehat{I\left(\mu_{2}\left|u_{2}\right|^{2} u_{2}+\beta\left|u_{1}\right|^{2} u_{2}\right)}\left(\eta_{1}\right) \widehat{I u_{1}}\left(\eta_{2}\right) \widehat{\overline{I u_{1}}}\left(\eta_{3}\right) \widehat{I u_{2}}\left(\eta_{4}\right) .
\end{aligned}
$$


By the results of [6], we know that

$$
\begin{aligned}
& \left|\int_{0}^{\delta} \int_{\sum_{j=1}^{4} \zeta_{j}=0}\left(1-\frac{M\left(\zeta_{2}+\zeta_{3}+\zeta_{4}\right)}{M\left(\zeta_{2}\right) M\left(\zeta_{3}\right) M\left(\zeta_{4}\right)}\right) \widehat{\widehat{\phi_{1}}}\left(\zeta_{1}\right) \widehat{\phi_{2}}\left(\zeta_{2}\right) \widehat{\bar{\phi}_{3}}\left(\zeta_{3}\right) \widehat{\phi_{4}}\left(\zeta_{4}\right)\right| \\
& \quad \leq C N^{-1} C\left(N_{1}, N_{2}, N_{3}, N_{4}\right)\left\|\phi_{1}\right\|_{X_{-1, \frac{1}{2}+}} \prod_{j=2}^{4}\left\|\nabla \phi_{j}\right\|_{X_{0, \frac{1}{2}+}}
\end{aligned}
$$

for sufficiently small $C\left(N_{1}, N_{2}, N_{3}, N_{4}\right)$ and any functions $\phi_{j}(j=1, \ldots, 4)$ with positive spatial Fourier transforms supported on

$$
\langle\xi\rangle \sim 2^{k_{j}} \equiv N_{j}
$$

for some $k_{j} \in\{0,1, \ldots\}$.

Letting $\phi_{1}=\Delta I u_{k}(k=1,2), \phi_{j}=I u_{k}(j=2,3,4, k=1,2)$ in (80), we obtain

$$
\operatorname{Term}_{1}+\operatorname{Term}_{3}+\operatorname{Term}_{5}+\operatorname{Term}_{7} \leq C N^{-1} .
$$

Now we need to estimate Term 2, Term $_{4}$, Term $_{6}$, Term $_{8}$.

First we consider Term $_{2}$. It is easy to see that $\mid$ Term $_{2}|\leq| \operatorname{Term}_{21}|+| \operatorname{Term}_{22} \mid$ with

$$
\begin{aligned}
\operatorname{Term}_{21} & =\int_{0}^{\delta} \int_{\sum_{j=1}^{4} \zeta_{j}=0} \mu_{1} L_{1}(\zeta) \overline{I\left(\mu_{1}\left|u_{1}\right|^{2} u_{1}\right)}\left(\zeta_{1}\right) \widehat{I u_{1}}\left(\zeta_{2}\right) \widehat{I u_{1}}\left(\zeta_{3}\right) \widehat{I u_{1}}\left(\zeta_{4}\right), \\
\operatorname{Term}_{22} & =\int_{0}^{\delta} \int_{\sum_{j=1}^{4} \zeta_{j}=0} \mu_{1} L_{1}(\zeta) \widehat{I\left(\beta\left|u_{2}\right|^{2} u_{1}\right)}\left(\zeta_{1}\right) \widehat{I u_{1}}\left(\zeta_{2}\right) \widehat{I u_{1}}\left(\zeta_{3}\right) \widehat{I u_{1}}\left(\zeta_{4}\right)
\end{aligned}
$$

By the results of [6], we know that

$$
\begin{aligned}
& \left|\int_{0}^{\delta} \int_{\sum_{j=1}^{6} \zeta_{j}=0} L_{1}(\zeta) P_{N_{123}} \widehat{I\left(\phi_{1} \phi_{2} \phi_{3}\right)}\left(\zeta_{1}+\zeta_{2}+\zeta_{3}\right) \widehat{I \phi_{4}}\left(\zeta_{4}\right) \widehat{\overline{I\left(\phi_{5}\right)}}\left(\zeta_{5}\right) \widehat{I \phi_{6}}\left(\zeta_{6}\right)\right| \\
& \quad \leq C N^{-\frac{3}{2}} N_{4}^{0-} \prod_{j=1}^{6}\left\|I \phi_{j}\right\|_{X_{1, \frac{1}{2}+}}
\end{aligned}
$$

where $0 \leq \hat{\phi}_{j}$ is supported for $\left|\xi_{j}\right| \sim N_{j}=2^{k_{j}}$ and

$$
N_{4} \geq N_{5} \geq N_{6} \quad \text { and } N_{4} \geq C N,
$$

while let $P_{N_{123}}$ be the projection onto functions supported in the $N_{123}$ dyadic spatial frequency shell.

Letting $\phi_{j}=u_{1}(j=1,2, \ldots, 6)$ in Term 21 and $\phi_{1}=\phi_{2}=u_{2}, \phi_{j}=u_{1}(j=3,4,5,6)$ in Term $_{22}$, using (83) and proceeding as in [6], we can obtain

$$
\left|\operatorname{Term}_{2}\right| \leq C N^{-1} \text {. }
$$
proved.

Similarly, we can get the bounds for Term 4, Term $_{6}$, Term $_{8}$. Proposition 2 is

ACKNOwledgement. The authors would like to thank the unknown referee very much for many valuable suggestions which improve the presentation of the paper. The 
research is partially supported by the National Natural Science Foundation of China 10631020 and SRFDP 20060003002.

\section{REFERENCES}

1. N. Akhmediev and A. Ankiewicz, Partially coherent solitons on a finite background, Phys. Rev. Lett. 82 (1999), 2661.

2. J. Bourgain, Refinements of Strichartz's inequality and applications to 2D-NLS with critical nonlinearity, Int. Math. Res. Notices 5 (1998), 253-283.

3. J. Bourgain, Global solutions of nonlinear Schrödinger equations (American Mathematical Society, Providence, RI, 1999).

4. H. Buljan, T. Schwartz, M. Segev, M. Soljacic and D. Christoudoulides, Polychromatic partially spatially incoherent solitons in a noninstantaneous Kerr nonlinear medium, J. Opt. Soc. Am. B 21 (2004), 397-404.

5. T. Cazenave and F. Weissler, The Cauchy problem for the nonlinear Schrödinger equation in $H^{1}$, Manuscripta Math. 61 (1988), 477-494.

6. J. Colliander, M. Keel, G. Staffilani, H. Takaoka and T. Tao, Almost conservation laws and global rough solutions to a nonlinear Schrödinger equation, Math. Res. Lett. 9 (2002), 659-682.

7. J. Colliander, M. Keel, G. Staffilani, H. Takaoka and T. Tao, Multi-linear estimates for periodic Kdv equations, and applications, J. Funct. Anal. 211 (2004), 173-218.

8. J. Colliander, S. Raynor, C. Sulem and J. D. Wright, Ground state mass concentration in the $L^{2}$-critical nonlinear Schrödinger equation below $H^{1}$, Math. Res. Lett. 12 (2005), 357-375.

9. B. Gidas, W. M. Ni and L. Nirenberg, Symmetry of positive solutions of nonlinear elliptic equations in $\mathbb{R}^{n}$, Adv. Math. Studies 7 (1981), 369-402.

10. F. T. Hioe, Solitary waves for $N$ coupled nonlinear Schrödinger equations, Phys. Rev. Lett. 82 (1999), 1152-1155.

11. L. Ma and L. Zhao, Sharp thresholds of blow-up and global existence for the coupled nonlinear Schrödinger system, J. Math. Phys. 49 (2008), 062103.

12. L. Ma and L. Zhao, Uniqueness of ground state of some coupled nonlinear Schrödinger system, J. Diff. Eq. 245 (2008), 2551-2565.

13. L. Ma and L. Zhao, On energy stability for the coupled nonlinear Schrödinger system (Zeitschrift fur Angewandte Mathematik und Physik, 2009).

14. H. Takaoka, Global well-posedness for the Schrödinger equations with derivative in a nonlinear term and data in low order Sobolev space, Electronic J. Diff. Eq. 42 (2001), 1-23.

15. K. Yajima, Existence of solutions for Schrödinger evolution equations, Comm. Math. Phys. 110 (1987), 415-426. 